\title{
A Program to Treat Hepatitis B in North Korea: A Model of Antiviral Therapy in a Resource-Poor Setting
}

\author{
Alice Unah Lee ${ }^{1,2}$, Heidi Linton ${ }^{3}$, Marcia Kilsby ${ }^{4}$, and David C. Hilmers ${ }^{2,5}$ \\ ${ }^{1}$ Department of Gastroenterology and Liver Services, Concord Repatriation General Hospital, University of Sydney, ${ }^{2}$ Hepatitis B Free, Sydney, \\ Australia, ${ }^{3}$ Christian Friends of Korea, Black Mountain, NC, ${ }^{4}$ Global Care Partners, Berrien Springs, MI, and ${ }^{5}$ Department of Internal Medicine \\ and Pediatrics, Center for Space Medicine, Baylor College of Medicine, Houston, TX, USA
}

Despite the well-proven, safe and effective therapies for hepatitis B infection, delivery of treatment remains a significant challenge in resource-poor settings. Geopolitical and economic restrictions present additional difficulties in providing care in North Korea. However, treatment of patients with chronic hepatitis B remains a top priority for both the North Korean Ministry of Public Health and international agencies working in North Korean hepatitis healthcare facilities. Working in partnership, a path was created to institute this much-needed program. A consortium of United States and Australian humanitarian non-governmental organizations along with generous individual and corporate donors working in concert with local and national health authorities have succeeded in establishing the first hepatitis B treatment program in North Korea. The essential elements of this program include renovation of existing hepatitis hospitals, access to antiviral medications, establishment of laboratory facilities, creation of medical documentation and record-keeping, training of local health care professionals, and quarterly visits by international volunteer physicians and laboratory experts. Management and treatment decisions are made bilaterally. To date, nearly 1,500 patients have been evaluated, and over 800 have been started on long-term antiviral therapy. It is envisioned that this program will eventually be managed and funded by the Democratic People's Republic of Korea Ministry of Public Health. This program's success demonstrates a potential model for delivery of antiviral therapy for patients suffering from hepatitis B in other developing countries. (Gut Liver 2018;12:615-622)

Key Words: Hepatitis B; Antiviral therapy; Democratic People's Republic of Korea; HOPE Program; Cirrhosis

\section{INTRODUCTION}

Lost among the tensions on the Korean Peninsula over the past years has been the continued suffering of 25 million ordinary North Korean citizens, many who bear untold hardship from malnutrition, natural disasters, and disease. Economic and political challenges have reduced the number of international aid agencies providing assistance and have limited the scale of their efforts. Such work not only provides much needed support but represents a model of ongoing cooperation and understanding during difficult times. We describe how Christian Friends of Korea (CFK), a non-governmental organization (NGO) based in the United States works alongside the North Korean Ministry of Public Health (MoPH) to fight diseases such as chronic hepatitis B (CHB), treating patients and advocating for their needs. This partnership shows how cooperation and good-will can be achieved while positively impacting the health of its citizens.

Apart from the obvious political tensions, tightened US and United Nations (UN) sanctions in 2017 have effectively stopped most humanitarian work. Special licenses from the U.S. Departments of Treasury and Commerce are required for the procurement and shipping of all goods by American NGOs. UN sanctions adopted in December 2017 now prohibit any metal (including medical supplies such as phlebotomy needles) from being delivered to the Democratic People's Republic of Korea (DPRK), requiring NGOs to appeal to the UN Sanctions Committee for exemptions. Enhanced Chinese customs restrictions prohibit many critical goods required for humanitarian engagement; wire transfers to longtime Chinese suppliers engaged in procurement activities on behalf of NGOs are now being blocked; and third-party vendors (banks, suppliers, shipping companies, etc.) scrutinize transactions or refuse to do business

Correspondence to: Alice Unah Lee

Department of Gastroenterology and Liver Services, Concord Repatriation General Hospital, University of Sydney, Hospital Road, Concord NSW 2139, Australia

Tel: +61-412133131, Fax: +61-297676767, E-mail: aliceulee@gmail.com Received on March 9, 2018. Revised on May 29, 2018. Accepted on June 7, 2018. Published online August 30, 2018 pISSN 1976-2283 eISSN 2005-1212 https://doi.org/10.5009/gnl18115

@) This is an Open Access article distributed under the terms of the Creative Commons Attribution Non-Commercial License (http://creativecommons.org/licenses/by-nc/4.0) which permits unrestricted non-commercial use, distribution, and reproduction in any medium, provided the original work is properly cited. 
with NGOs out of fear of violating sanctions, even when licenses are in place. Containers of relief supplies have been held up in ports for lengthy periods while custom officials work through the legal complexity of new sanctions, leading to delays in shipping, additional costs and program setbacks.

The obstacles faced by foreign aid agencies working in the DPRK, while always daunting, are now enormous. Nearly every aspect of providing modern medical care is fraught with difficulty. Hospitals and care centers need renovation, clean water, reliable power, and medications for hepatitis have not been available. Laboratory facilities are outdated and many lack equipment and reagents. While there is no shortage of physicians in the DPRK, many have restricted access to updated information. Visits by foreign partners must be vetted across multiple governmental entities, and logistics organized and coordinated with local, provincial and central authorities. American humanitarian aid workers are now required to apply to the U.S. Department of State for an exemption from the travel ban for each visit that they make. MoPH personnel accompany foreign visitors, yet they, too, are understaffed, limiting the personnel and visits that can be facilitated each year. Those from other countries are often reluctant to volunteer under the current uncertain security environment. Patients themselves are uncertain as to what to expect and can be wary of foreign (particularly American) health care providers, at least at the onset.

Outside of UN and World Health Organization (WHO)supported work which is currently underfunded, most external medical aid programs in North Korea are undertaken by a handful of NGOs (with limited staffing and budgets) that have pursued principled engagement, some for two decades or more. More than anywhere else in the world, trust and partnership between NGOs, local authorities and the MoPH remain critical.

For many years the MoPH had identified hepatitis B as a priority disease in the DPRK and made requests to NGOs to address shortfalls in all aspects of hepatitis B care. Prevalence rates are likely to reflect those of similar countries of comparable background and are likely to be high. ${ }^{1}$ Vaccination gaps in children had been addressed as part of an Expanded Program of Immunization in 2003 for infants and children and a catchup vaccination program took place in 2009. ${ }^{2}$ A further 7 million children were vaccinated between 2010 and 2012. However, most adults and at-risk individuals such as health care workers and close contacts of infected individuals remain untested and unvaccinated. Furthermore, antiviral treatment programs to care for those with $\mathrm{CHB}$ were essentially non-existent. Requests for antiviral medications and laboratory capacity came repeatedly from local doctors during visits by volunteers from CFK who were checking on clean water projects or deliveries of blankets, greenhouses, and tractors at health care facilities. While prevention remains the key to controlling this epidemic, the daunting task of treating those with $\mathrm{CHB}$ was a challenge that could not be ignored.

\section{THE HOPE PROGRAM}

Antiviral therapy for hepatitis B has been available for over two decades in settings where its high cost is affordable. However, even in wealthy countries, only a minority of those who need therapy are actually receiving it. Limited amounts of these medications are now reaching those who live in less developed nations where the disease burden is the greatest. To date, the challenges in delivering antiviral therapy for hepatitis B have been considered too onerous to overcome and hence, largescale treatment programs have been left off the table. In contrast, highly effective human immunodeficiency virus therapy is widely available in the most resource-challenged settings which raises the question as to why hepatitis B treatment (which often includes the same medications) should not be equally accessible.

The WHO recognized this incongruence and in 2015 published global guidelines for the prevention, care and treatment of CHB, with resource-limited regions in mind (see a summary in Table 1). ${ }^{3}$ These guidelines simplified the algorithm for treatment while taking into account budgetary constraints and limited availability of laboratory investigations. Point of care (POC) kits for key hepatitis tests, some with sensitivities and specificities approaching traditional laboratory-based tests are affordable and widely available. Treatment decisions with the new guidelines can be made in the absence of viral load testing. Thereby, a new pathway for the treatment of hepatitis B opened in the DPRK and other regions of the world with a high prevalence of CHB but limited resources.

In order to implement such a project in the DPRK, essential requirements included access to patients, medical personnel, hospitals, medications, ultrasound, and laboratory services. An alliance of NGOs and generous donors was formed to provide CFK with additional expertise and resources (Table 2). CFK has been working in the DPRK since 1995, providing aid after floods and other natural disasters, delivering food and medicines at health-care facilities and rest homes, and engaging in capacitybuilding projects in technical areas such as agriculture, clean water, renewable energy, infrastructure, tuberculosis diagnostics and education. It has established important contacts within the $\mathrm{MoPH}$ and with local and regional government officials. An Australian NGO, Hepatitis B Free (HBF), was formed to facilitate prevention of and delivery of care for hepatitis B in areas with limited access to healthcare. Global Care Partners (GCP) is an American NGO that focuses on the improvement of clinical laboratory diagnostic services in resource-challenged countries through on-site education and training, consultation, laboratory assessment and design, and procurement of appropriate laboratory equipment. All three organizations came together to work with the MoPH to initiate a pilot program to provide hepatitis B care to patients in the DPRK.

A memorandum of understanding (MOU) between the program partners and the MoPH was negotiated, and a program 
Table 1. Summary of WHO Recommendations for Persons with CHB When Viral Load Testing Is Not Available

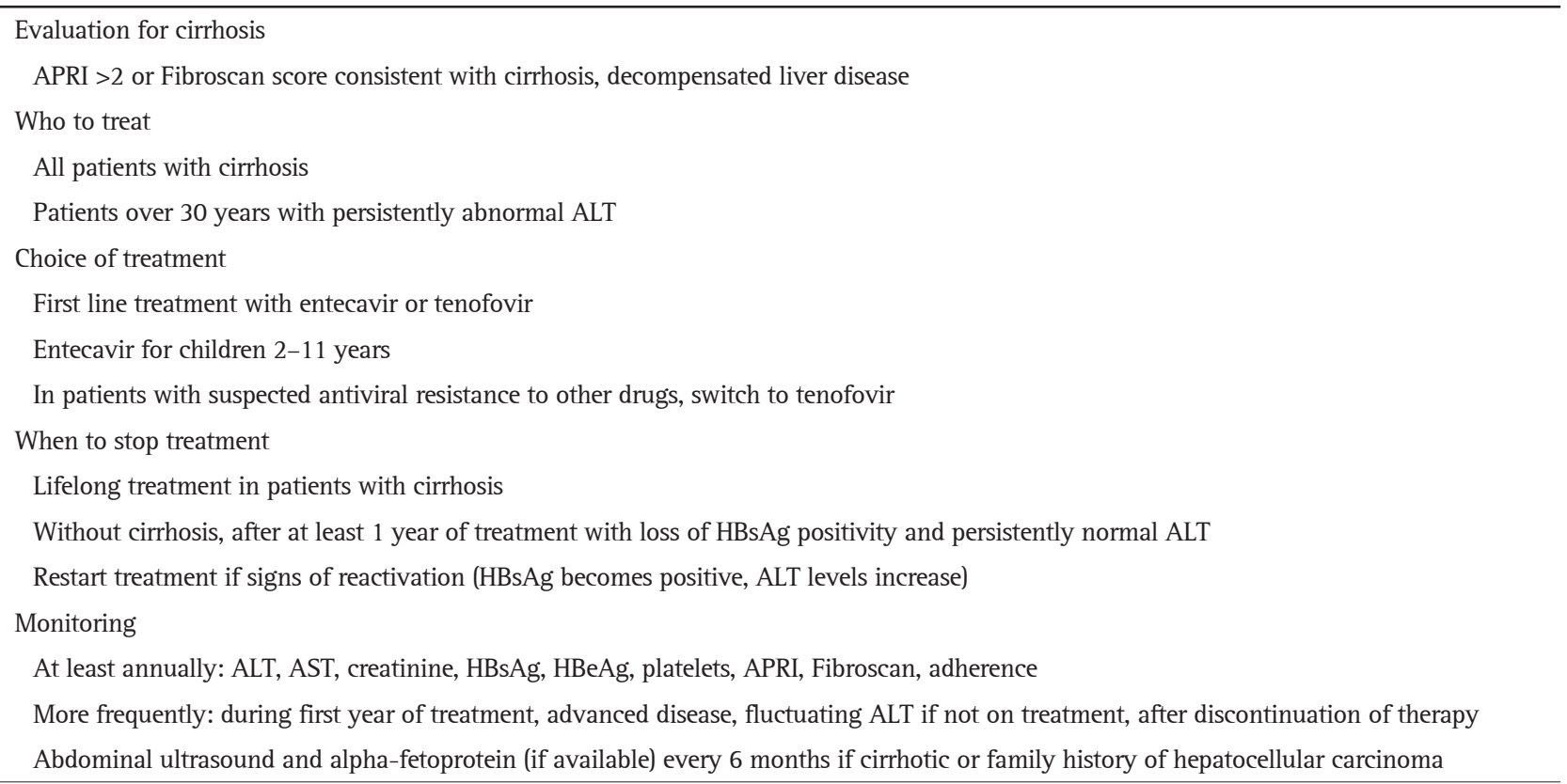
WHO, World Health Organization; CHB, chronic hepatitis B; APRI, aspartate aminotransferase (AST) to platelet ratio index; ALT, alanine aminotransferase; HBsAg, hepatitis B surface antigen; HBeAg, hepatitis B e antigen.

Table 2. Contributions of NGO in HOPE

\begin{tabular}{l}
\hline Christian Friends of Korea (CFK), Black Mountain, NC, USA \\
Overall coordination of program with MoPH and US authorities \\
Infrastructure development (water, electricity, building construc- \\
tion) \\
Import licenses, transport and storage of supplies and medications \\
Maintenance of patient database and medical records \\
Hepatitis B Free (HBF), Sydney, Australia \\
Overall responsibility for treatment of hepatitis patients \\
Acquisition of antivirals \\
Patient medical records \\
Recruitment of volunteer physicians \\
Training of local physicians \\
Global Care Partners (GCP), Berrien Springs, MI, USA \\
Overall responsibility for clinical diagnostic testing of patients' \\
specimens \\
Design of laboratory facilities \\
Acquisition of laboratory supplies and analyzers \\
Training of local laboratory personnel
\end{tabular}

NGO, non-governmental organization; HOPE, Hepatitis B Overview and Program to Treat; MoPH, Hepatitis B Overview and Program to Treat.

protocol document called HOPE (Hepatitis B Overview and Program to Treat) was created. The MOU delineated the responsibilities of the MoPH and NGOs and the objectives of the program. The primary aim was to: “...provide antiviral therapy for treatment of eligible patients in the DPRK, to improve patient outcomes and reduce morbidity and mortality from hepatitis $B$ related illnesses through a program of testing, treatment and follow-up." Secondary objectives included the development of preventative strategies to reduce new infections, to increase screening and case identification with linkage to treatment, and to provide education and training. The duration of the program was envisioned to be 10 years with a goal of treating 5,000 patients with $\mathrm{CHB}$. If the number of patients on treatment exceeds this number, appeals for new donations will be made. At the end of this period, the objective is that the program will be selfsustaining with local physicians and the MoPH fully capable of carrying on the program without outside assistance.

\section{INFRASTRUCTURE DEVELOPMENT}

The two initial sites selected for the pilot program were the Pyongyang \#2 (Hepatitis) Prevention Hospital and Kaesong \#2 (Hepatitis) Prevention Hospital. Pyongyang \#2 Prevention Hospital, located in the capital, is the referral center for patients with hepatitis in Pyongyang City. The hospital has a laboratory, pharmacy and Korean traditional medicine department and supports both inpatient and outpatient care. Therapeutic and diagnostic endoscopic procedures (fewer than 20 per year) are offered when equipment is working, and consumables such as variceal band ligators are available through donations. The hospital is connected to grid power, but due to inconsistent power supply, a solar/battery array with a backup generator often serves as the main power source. The second site is Kae- 
song \#2 (Hepatitis) Prevention Hospital, situated in the outskirts of Kaesong City, $174 \mathrm{~km}$ south of Pyongyang. The hospital is comprised of several one-story buildings with both inpatient and outpatient care facilities. It serves as the referral center for patients with hepatitis in Kaesong City. There was no centrally distributed power or water available at this facility when the project first began. A third site in the coastal city of Haeju in southwest North Korea is under renovation, and the first cohort of patients was recently screened for treatment. A fourth site has also been identified and funding sources are being pursued.

The first and most difficult challenge was to create laboratory infrastructure, but significant work had already begun. Since 2008, GCP has been collaborating with CFK in the DPRK assisting with planning, renovating, and equipping the National Tuberculosis Reference Laboratory (NRL) in Pyongyang as tuberculosis remains the other major public health burden in the DPRK. ${ }^{4}$ GCP helped create a clinical laboratory concurrent with the development of the tuberculosis-specific diagnostics at the NRL. The clinical lab provided hematology, chemistry, immunology, and body fluid diagnostics, and GCP supported bi-annual training for the staff to develop their skills. The laboratory professionals who trained at Pyongyang now serve as instructors at the newer sites. Important additions to the laboratory capability which were facilitated by GCP included the provision of hematology and biochemistry analyzers. ${ }^{5}$

CFK has substantial technical expertise in the construction and renovation of buildings, including the NRL, as well as the provision of clean water and power. Using this experience base, two solar-powered, modern clinical labs with newly drilled wells at the pilot sites in Pyongyang and Kaesong were built and equipped, supported by private fundraising and volunteers from CFK, HBF, and GCP, who worked closely with local hospital staff. The laboratory in Pyongyang was recently designated as the National Hepatitis Reference Laboratory.

\section{TREATMENT}

Review of priority patients for therapy started in late fall, 2015, and treatment protocols were developed based on WHO guidelines, with some adjustment made for the local context. Each aspect of this program was negotiated in detail with the local partners. Negotiations at times were delicate, and patience was constantly tested on both sides. Screening and confirmation that patients were hepatitis B surface antigen positive was the first step. Patients over the age of 18 were considered eligible. Those between 3 and 17 years old were enrolled after discussion with a pediatrician and the patient's guardian. Eligibility criteria for treatment were consistent with WHO and recognized international hepatology guidelines. Treatment exclusion criteria include known sensitivity to treatment drugs, life expectancy of less than 6 months from any cause, and inability to comply with the treatment protocol (Table 3). All patients with $\mathrm{CHB}$ and cirrhosis are deemed highest priority for therapy. In those without cirrhosis, persistent elevation in alanine aminotransferase (ALT) and risk of progression of liver disease are considerations to initiate treatment (Fig. 1). All patients are asked to sign an agreement to comply with each program requirement including periodic diagnostic testing, attendance for follow-up visits and compliance with medications. Country-specific patient educational resources were prepared in Korean and approved by local authorities for use.

The program requires that both a qualified international physician and a locally trained physician assess each potential patient prior to initiating treatment. This evaluation includes clinical history, physical examination and careful assessment of laboratory and radiologic investigations. Clinical laboratory assessment includes confirmation of hepatitis B status using POC tests for hepatitis B surface antigen (HBsAg), surface antibody (HBsAb), e antigen (HBeAg), and hepatitis C antibody (HCV Ab). Both SD Bioline (SD Bioline Seoul, South Korea; Alere Diagnostics, Waltham, MA USA) and ABON (ABON Biopharm, Hangzhou, China) POC tests were selected to ensure access to more than one supply source (see Table 4). Biochemical parameters include a complete blood count (CBC) as well as analyzer measurements of aspartate aminotransferase (AST), ALT, and creatinine $(\mathrm{Cr})$. With these values a calculation of the AST to platelet ratio index can be made to determine eligibility for treatment, as indicated in the WHO guidelines. Negotiations are currently underway to add other tests such as quantitative hepatitis B DNA viral load, but ongoing costs in supplying the lab currently limit the addition of new capabilities and staff training. Selection of the analyzers was based on equipment reliability and availability of inexpensive, high-quality consumables. An ultrasound is performed on each patient looking for signs of cirrhosis, ascites, splenomegaly, and portal hypertension as well as surveillance for liver masses, both benign and malignant. All ultrasound equipment was donated. Additionally, a Fibroscan ${ }^{\mathrm{TM}}$ (Echosense, Paris, France) machine was provided by a generous donor and has been invaluable in staging liver disease and guiding treatment decisions. Based on history, physical examination, lab

Table 3. Inclusion and Exclusion Criteria

Inclusion criteria
Hepatitis B surface antigen positive
Age 18 or above (ages $3-17$ if approved by pediatrician and guard-
ian)
Exclusion criteria
Inability to comply with program requirements (non-compliance
with medications and clinic visits, continued alcohol abuse, re-
fusal of lab testing)
Life expectancy <6 months
Known allergy to or intolerance of drugs




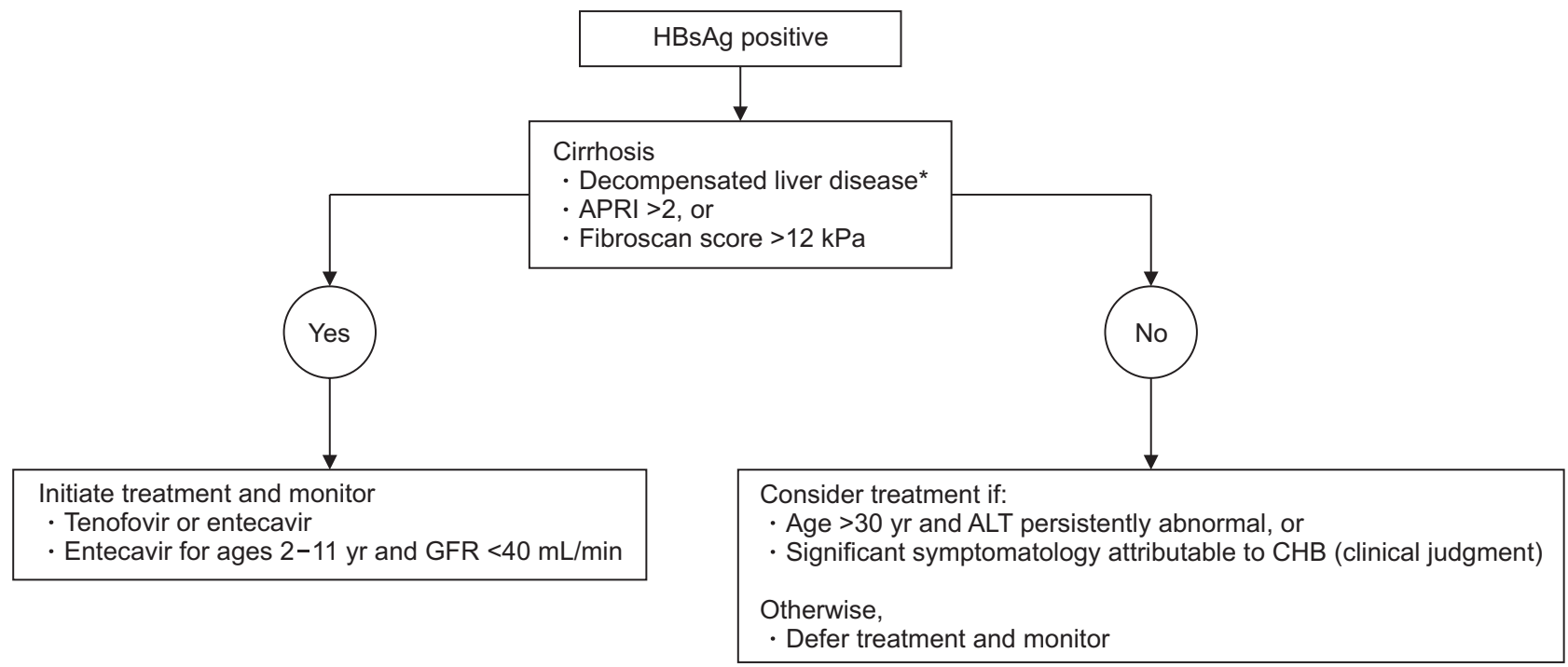

Fig. 1. Simplified guidelines for selection of patients to undergo treatment.

HBsAg, hepatitis B surface antigen; APRI, aspartate aminotransferase to platelet ratio index; GRF, glomerular filtration rate; ALT, alanine aminotransferase; CHB, chronic hepatitis B. *Clinical signs and symptoms of decompensated cirrhosis include ascites, variceal bleeding, hepatic encephalopathy, jaundice, extrahepatic symptoms. Adapted from World Health Organization (WHO) treatment guidelines, 2015.

Table 4. Point of Care Hepatitis Test Characteristics

\author{
SD Bioline HBsAg Rapid Test \\ SD Bioline Seoul, South Korea/Alere Diagnostics, Waltham, MA
} USA

Advertised sensitivity/specificity: 100\%/100\%

https://www.alere.com/en/home/product-details/sd-bioline-hbsag. html

ABON Hepatitis B Combo Test (HBsAg, HBsAb, HBeAg, HBeAb, $\mathrm{HBcAb)}$

ABON Biopharm, Hangzhou, China

Advertised sensitivity/specificity: 99.0\%/96.8\%

http://perufreelo.com/deuce/wp-content/uploads/2016/02/ab2. pdf

SD Bioline HCV IgG Rapid Test

SD Bioline Seoul, South Korea/Alere Diagnostics, Waltham, MA USA

Advertised sensitivity/specificity: 100\%/99.4\%

http://gms-world.com/sc001/MISdata/MIS/SD\%20MIS/HCV/HCV. pdf

$\mathrm{HBsAg}$, hepatitis B surface antigen; HBsAb, hepatitis B surface antibody; HBeAg, hepatitis B e antigen; HBeAb, hepatitis B e antibody; $\mathrm{HBcAb}$, hepatitis B core antibody.

results, ultrasound and Fibroscan ${ }^{\mathrm{TM}}$ patients are prioritized for treatment per WHO guidelines (Fig. 1). A goal of this program has been to maintain cost efficiency without compromise to patient care.

Patient access to life-long antiviral therapy in a country where programmatic transition to local health authorities is uncertain was a significant challenge. The first identified priority was to find a way to procure medicines for the DPRK and to establish a pathway for medicines to be imported into a country that was subject to sanctions. After many months of negotiations including extensive legal counsel to ensure compliance with international sanctions, a donor commitment for the first cohort of patients for therapy was confirmed. This continues to be a work in progress with the need for constant reevaluation as program requirements and limitations on imports change. The most effective antivirals for CHB, tenofovir and entecavir, were ordered and arrived in the summer of 2016, just in time to start a small cohort of the sickest patients on treatment. To date nearly 1,500 patients have been screened by teams of international volunteer physicians, and over 800 have started antiviral therapy (see Table 5 for baseline characteristics). External team visits occur four times per year and patients are seen at least twice annually. Each visit includes interval history, physical examination, lab testing (ALT, Cr, platelets) and ultrasound for liver cancer screening. Annual HBsAg testing is done, and Fibroscan is performed on a bi-annual basis to document disease progression or regression. Patients are counseled, and compliance is reinforced.

Clinical reviews are accomplished on each patient with local and international doctors working side by side; treatment decisions are made jointly, often after prolonged discussions. Skills transfer occurs bilaterally across all elements of the project. In time this program will belong to the local healthcare workers, and it is essential to empower them with all the tools needed to take full ownership. Nearly all patients have been seen in follow-up, many with a new lightness in their hearts and with hope for a brighter future. Approximately 30 of the sickest 
Table 5. Characteristics at Baseline of Patients on Treatment

\begin{tabular}{lcc}
\hline \multicolumn{1}{c}{ Clinic } & Pyongyang & Kaesong \\
\hline Total patients on treatment & 604 & 249 \\
Age, yr* & $43.82(13-71)$ & $44.81(19-65)$ \\
Sex, male/female & $426 / 178$ & $170 / 79$ \\
Fibroscan score, $\mathrm{kPa}$ & $16.1(3.5-75)$ & $17.8(3.5-75)$ \\
APRI & $2.32(0.10-56.0)$ & $2.01(0.27-28.88)$ \\
Platelets, $\times 10^{9} / \mathrm{L}$ & $118(12-358)$ & $124(19-648)$ \\
Cirrhotics, $\%$ of total by Fibroscan ${ }^{\dagger}$ & 63.4 & 69.2 \\
Cirrhotics, \% of total by APRI ${ }^{\dagger}$ & 57.0 & 50.2 \\
ALT, IU/L & $59.8(5.0-955.0)$ & $53.9(12.0-988.4)$ \\
AST, IU/L & $64.0(5.0-1,373.0)$ & $60.6(18.0-493.5)$ \\
Patients on antivirals, tenofovir/entecavir & $470 / 134$ & $197 / 52$ \\
\hline
\end{tabular}

Data are presented as number or median (range).

APRI, aspartate aminotransferase (AST) to platelet ratio index; ALT, alanine aminotransferase.

${ }^{*}$ Mean (range); ${ }^{\dagger}$ Fibroscan score $\geq 12.0 \mathrm{kPa} ;{ }^{\ddagger} \mathrm{APRI} \geq 2.0$.

patients have died despite starting therapy. Although records are not available which show the exact cause of death, it is presumed that they died from complications of end-stage liver disease. Others remain critically ill including a young mother who decompensated during child birth and now hopes that treatment will give her a chance to see her daughter grow to adulthood, and young men with new diagnoses of liver cancer which remains universally fatal in the DPRK.

\section{DRUG DISPENSING AND ACCOUNTABILITY}

For the integrity of the program, a precise accounting of all medicines donated is absolutely essential. There are four stages of accountability of antiviral medications supplies including: receipt in country, onward shipping to treatment centers, treatment center receipt, and patient dispensing and accountability. On arrival in country at the central depot, each shipment of antiviral drugs must be recorded on the drug shipment receipt $\log$ as received and a physical count taken. Onward shipments to treatment centers from the central depot are recorded on shipping logs located at the clinics, and another physical count is taken. Each treatment center must have its own individual folder containing its shipment receipt logs which are kept under separate physical control from the pharmacist who manages the individual patient dispensing and accountability logs. Each treatment center has its own drug shipment receipt log to document when antiviral medications arrive on site.

\section{PROGRAM DOCUMENTATION}

An electronic medical record system utilizing bar code scanning has been developed, despite the lack of internet connectivity or grid power. It became apparent after the first clinic visits that using paper records would soon become untenable as the program expanded. Lab results had to be manually entered into the Microsoft Access data base that was developed for the HOPE program. Each document generated during the clinic visit would need to be scanned at the end of the work day, and this became overly time-intensive for the team, even with only 100 patients per clinic day. After much discussion, a decision was made to develop an ad hoc system utilizing bar codes and fillable clinic forms that could be saved on laptop computers.

At the heart of the electronic system is a Microsoft Access data base where the master files reside. Upon entry into the program a patient is photographed with their unique patient number that is imprinted onto an identification card with a computer-generated bar code. Bar code labels are printed and affixed to the sample collection tubes when the patient has blood drawn. These tubes are sent to the lab and the bar code scanner on the lab analyzer reads the patient's identification number and automatically stores the lab results in its internal memory. The lab values can later be downloaded from the analyzer as an Access file and uploaded into the main data base.

Meanwhile, each physician brings a laptop to clinic and attaches a barcode scanner to the computer. Fillable forms using Microsoft Word with drop-down menus and click boxes have been developed for the initial visit and follow-up appointments. These documents are password protected. When a patient arrives at the examination station, his/her identification is confirmed, the identification card is scanned and the corresponding demographic information and lab values automatically populate the encounter forms. The provider also has access to all previous clinic visits and lab values through the Access data base. The visit can be efficiently documented with a series of clicks and a typed impression at the end of the form. At the end of the clinic day, each provider uploads the saved files onto a USB drive, the content of which is placed in the master data base, eliminating the need to scan or copy paper forms. This system has worked 
well without the need for a local area network or internet and has allowed the clinic to expand without being inundated by paper documentation. Future challenges will be to train local providers to use this system, to provide hardware, such as laptops, for them to use during clinic visits, and to maintain quality control.

\section{TRAINING, ADVOCACY, AND EDUCATION}

A critical component of the program is training local physicians, nurses, lab technicians, and data managers in order to insure sustainability. Lectures, demonstrations, and hands-on training are an integral part of laboratory training. Each time one of the clinic sites is visited, the international physicians provide in-depth lectures on the prevention and treatment of hepatitis and associated complications to local staff. This training has been very well-received with increased bilateral understanding of the requirements and barriers to improved care. Training of local staff continues to remain a challenge as travel within the DPRK by health professionals is discouraged, and time and cost constraints on international instructors often limit the number of sites that can be visited. Even when the team is in country, access to the health care workers for training is not always possible given the competing demands for their time.

Advocacy is critical to address current gaps and opportunities in hepatitis services, to raise awareness about the burden of hepatitis in DPRK, and to enable long-term sustainability for the program, including funding and policy. Promotion of preventative strategies is essential including vaccination of highrisk groups, interruption of mother to child transmission, and decreasing healthcare-transmitted infections (including injection safety, universal precautions, and blood bank safety). Decisionmakers must be encouraged to enact policies that enable improvement of hepatitis services. In addition to the MoPH, regular meetings have been held with in-country stakeholders such as WHO, United Nations Children's Fund (UNICEF), and other NGOs to advocate for additional funding and awareness.

Education of the public, including affected patients and their families, is also a primary objective. Upon entrance into the program each patient receives a handbook that illustrates in easy to understand language some of the key principles of the disease, its prognosis, possible side effects of medications, caring for oneself, and preventing transmission. Information sheets are given to patients that describe the HOPE program and give more detail about the natural history of CHB. During each visit, patients are given ample opportunity to ask questions and to inquire about the results of their tests. Patients are encouraged to refer family members or friends from their neighborhoods or villages to the clinic for testing if their acquaintances have similar signs and symptoms. Many have done so already.

\section{FUTURE DIRECTIONS}

In working together to tackle a disease of staggering proportions in the DPRK, we have found ways to effectively navigate difficult waters, communicate and cooperate despite severe sanctions. Our experience has been invaluable in creating a model for treatment of $\mathrm{CHB}$ in other resource-poor regions with high burdens of disease. Already, our experience is being used to start similar programs in western Pacific islands such as Kiribati, some of which have the highest rates of CHB in the world. Ongoing advocacy to further reduce prices (in all elements of hepatitis care) and to provide funding and availability for hepatitis B treatment and prevention should be emphasized to insure equitable access to care throughout the world.

In the DPRK we have only begun to scratch the surface of the nation's hepatitis needs, and it is unlikely that our small team can make a significant dent in the overall burden of CHB. However, for the patients and their families we have met, it is an important start and a source of real hope. Despite significant barriers such as travel restrictions on volunteers and a vulnerable supply chain, we plan to move forward as long as it remains possible for us to enter the DPRK safely.

A third clinic site has been approved and funding obtained to establish a program at the South Hwanghae \#2 Prevention Hospital in Haeju, located in the southwestern region of the DPRK. Construction and renovation have been interrupted by enhanced sanctions but are expected to resume soon. The first patients were seen in Haeju during the first half of 2018, supported by the laboratory in Pyongyang. Expansion of lab capabilities to include viral load testing is envisioned to improve diagnostics for hepatitis B and to enable treatment of hepatitis C. Viral load testing would also facilitate treatment of pregnant women who are infected with CHB in accordance with recognized protocols; this would be an important milestone in the prevention of vertical transmission of hepatitis B. As we build capacity, each step forward becomes more complex for both visitors and local partners.

On a personal level we worry about the fate of our patients who have benefited from treatment and for those we have yet to meet. In a broader sense, we have witnessed the extraordinary value of a cooperative health program in cultivating good-will and understanding while positively impacting a serious health problem. We have felt the gratitude and genuine comradery that has developed between all of the partners and over 1,500 patients. We hear their appeals at the end of each visit for us to return soon.

\section{CONFLICTS OF INTEREST}

No potential conflict of interest relevant to this article was reported. 


\section{REFERENCES}

1. Lee D, Park SM. Cost-effectiveness analysis of hepatitis B vaccination strategies to prevent perinatal transmission in North Korea: selective vaccination vs. universal vaccination. PLoS One 2016;11:e0165879.

2. Unnewehr M, Stich A. Fighting hepatitis B in North Korea: feasibility of a Bi-modal prevention strategy. J Korean Med Sci 2015;30:1584-1588.

3. World Health Organization (WHO). Guidelines for the prevention, care and treatment of persons with chronic hepatitis B infection [Internet]. Geneva: WHO; c2015 [cited 2017 Jul 20]. Available from: http://www.who.int/hiv/pub/hepatitis/hepatitis-b-guidelines/en.

4. Lee YH, Yoon SJ, Kim YA, Yeom JW, Oh IH. Overview of the burden of diseases in North Korea. J Prev Med Public Health 2013;46:111-117.

5. Perry S, Gresham L, Linton H, Schoolnik G. Engaging North Korea on mutual interests in tuberculosis control. Korea Economic Institute Academic Paper Series 2011:6;1-13. 